\title{
Concurrent Detection of Linear and Angular Motion using a Single-Mass 6-axis Piezoelectric IMU
}

\author{
Hela Almabrouk ${ }^{1}$ \\ Université de Sfax \\ Ecole Nationale d’Ingénieurs de Sfax, Sfax Tunisia \\ Faculté des Sciences de Monastir \\ Université de Monastir, Monastir Tunisia \\ C2N, UMR 9001, Université Paris-Saclay \\ 91120 Palaiseau, France \\ Mohamed Hadj Said ${ }^{2}$ \\ Center for Research in Microelectronics and \\ Nanotechnology (CRMN) \\ Sousse Technopole \\ Novation City, Tunisia
}

\author{
Fares Tounsi ${ }^{3}$, Brahim Mezghani ${ }^{4}$ \\ Université de Sfax, Ecole Nationale d'Ingénieurs de Sfax \\ UR-METS, Sfax, Tunisia \\ Guillaume Agnus ${ }^{5}$ \\ Centre de Nanosciences et de Nanotechnologies (C2N) \\ UMR 9001, Université Paris-Saclay \\ 91120, Palaiseau, France \\ Yves Bernard ${ }^{6}$ \\ GeePs | Group of Electrical Engineering - Paris, CNRS, \\ CentraleSupélec, Université Paris-Saclay, Sorbonne \\ Université, 3 \& 11 rue Joliot-Curie, Plateau de Moulon, \\ 91192, Gif-sur-Yvette CEDEX, France
}

\begin{abstract}
This paper exhibits operating system and performances of a novel single-mass 6-axis Inertial Measurement Unit (IMU) using piezoelectric detection. The electronic processing circuitry for the concurrent detection of linear and angular motion is proposed. The IMU structure is based on the use of 2 rings, connected with eight electrodes, implemented on the top of a piezoelectric membrane used for both sense and drive modes. The four inner electrodes are used for components detection due to the direct piezoelectric effect, while the outer electrodes are used to generate the drive mode due to the reverse piezoelectric effect. Through finite element analysis, we show that linear accelerations generate an offset voltage on the sensing electrodes, while angular rates lead to a change in the amplitude of the initial AC signal caused by the drive mode. The present work represents an innovative design able to separate 6 motion data from signals using only 4 electrodes. The specific electronic circuitry for acceleration and angular rate data dissociation shows a very efficient method for signal separation since no leakage readout occurs in all six axes. Besides, other particular interest is that under no circumstances, angular outputs disturb or affect acceleration ones and vice versa. The evaluated sensitivities are $364 \mathrm{mV} / \mathrm{g}$ and $65.5 \mathrm{mV} / \mathrm{g}$ for in-plane and out-ofplane linear accelerations, respectively. Similarly, angular rates sensitivities are $2.59 \mathrm{mV} / \mathrm{rad} / \mathrm{s}$ and $522 \mathrm{mV} / \mathrm{rad} / \mathrm{s}$.
\end{abstract}

Keywords-Inertial measurement unit; piezoelectric detection; angular rate; linear acceleration; electronic circuitry

\section{INTRODUCTION}

Inertial sensors, including magnetometers, gyroscopes and accelerometers are widely demanded in various fields such as navigation, automotive industry, robotic and military domains [1-4]. Commonly, each of these sensors has typically three degrees of freedom to measure from the three-dimensional surrounding space. Inertial measurement units (IMU) integrate multiple miniature inertial sensors to obtain comprehensive inertial parameters of the moving object, including the attitude, position, and speed information [5]. This information comes from the measure of rotational and linear inertial data such as angular velocity and gravitational force. IMU devices are highly independent and less susceptible to outside interference with fast data updates and strong stability. These devices are groundbreaking sensors that have been extensively studied by various researchers [6-9]. The multi-axis motion detection mechanisms rely on various sensing types. It can be piezoelectric [10-11], thermal [12], capacitive [13], piezoresistive [14], etc. However, piezoelectric inertial sensors are being known to offer important advantages compared to their counterparts. In fact, they can be used for wide range of frequencies by offering a very large dynamic range. Indeed, this type of inertial sensor is suitable for low amplitude shock measurements providing a very low noise level [15]. Moreover, piezoelectric sensors are known by offering reduced power consumption.

Many recent inertial sensors design allow to achieve linear and/or angular accelerations measurements through a singlecore detecting element [16-18]. Tuning fork and disk resonating structures are among the most successful singlecore devices $[10,16,17]$. The tuning fork resonator relies on the piezoelectric effect for the drive mode excitation and uses either piezoelectric or capacitive effect for the detection. The tuning fork structure is known by its simplicity and easy assembly with the associated electronic detection circuit. Nevertheless, it presents a low sensitivity and uses indirect method to measure the deflection especially the capacitance detection [16]. The other structure is composed by a resonant slotted disk supported by a central cylindrical post and surrounded by capacitive electrodes used to force and sense vibration. The resonator disk has the advantages of providing high quality factor and thermal stability due to the low anchor 
loss, air damping effect (perfored disk) and thermo-elastic dissipation [19]. Moreover, the symmetrical propriety of the disk structure offers a high sensitivity [17, 20]. In addition, the resonant disk devotes a large area of multiple drive and sense electrodes so that it becomes easier to operate and measure signals, using this structure, compared to other inertial sensors [21].

Inertial measurement unit devices are widely used in various applications due to the important information that it can provide including acceleration and angular rate data of a movable object. However, because of the use of two separated blocks of sensors, one for accelerometer and other for angular rate sensor, several limitations bound IMU development which consist mainly in size, cost and energy consumption. Therefore, IMUs are being restricted to relative bulk applications and thus, although its importance, conventional IMU devices become unsuitable to consumer applications unless reducing devices size. In this frame, along this paper, we detail the design performances of a 6-axis single-mass piezoelectric inertial measurement unit using one single sensor instead of two. Considering the significant advantages related to resonant structures, we are proposing an emerging mesoscale piezoelectric 6-DOF design capable to simultaneously detect 3-axis angular rate and 3-axis acceleration components, using a single-core detecting element.

The proposed structure uses a cylindrical proof mass as an oscillator, attached to the bottom center of an anchored piezoelectric disk. This configuration can improve performances due to the use of a great modal effective mass compared to other resonator designs [10, 21]. The structure uses piezoelectric effect to drive and sense the 6-DOF (Degree of Freedom) which is an advantage compared to the tuning fork and disk resonator structures, which generally detects only angular motion components [17, 19]. Moreover, the present design employs only four electrodes for driving the seismic mass and four other ones for separating the 6-DOF outputs. Finite Element Modeling (FEM) is used to simulate the detection mechanism performances of both angular velocity and linear acceleration on our proposed piezoelectric design and we extract output signals of each inertial component are extracted. FEM simulations are performed using the COMSOL ${ }^{\circledR}$ Multiphysics software package. Moreover, we are interested in the design of the conditioning part of the detection system using Orcad-PSPICE software.

In this paper, a presentation of the studied IMU design and its operation system will be firstly presented before going through an analytical analysis of the system operation by building the drive and sense mode equations. Then, numerical investigation of both linear and angular rate detections will be detailed. Results in terms of sensitivity will be compared with other designs from literature. We are also interested in the presentation of the separation electronic circuitry from which signals relative to three-axis acceleration and three-axis angular rate inputs will be faithfully identified.

The main objective of this work is to design a 6-axis sensor with the corresponding electronic circuit able to distinguish acceleration data from angular velocity ones, from the same sensing electrodes. We consider signals extracted from FEM simulations as input data for the development of the electronic circuitry.

\section{Presentation of the IMU Design And Its System's OPERATION}

The main advantage of the novel developed IMU sensor is the simultaneous detection of the 6-DOF inertial motions using a single seismic mass-based structure. The purpose and the challenge of the present work is the analysis of the mechanical IMU structure motion along with its corresponding electronic circuitry capable to separate acceleration from angular velocity data.

The proposed geometry of the 6-axis motion sensor is the millimeter range. The sensitive element, i.e. the piezoelectric membrane, is made of PZT material. It is basically formed by an external ring, a medium ring and an internal disk which are interconnected using attachments forming the sensitive part of the IMU design (Fig. 1a). The used structure is inspired from a 6-DoF IMU design proposed by Okada et al. [10] and it has been proved through previous works that our new proposed IMU design offers better performances [22, 23].

The shape of the flexible piezoelectric membrane has been carefully designed to generate maximum stress profiles in the sensitive parts, on which electrodes are placed, when subjected to any motions (linear accelerations and/or angular rate rotations). This PZT layer is superimposed on a circular diaphragm made of high precision Elinvar which is an alloy that has low thermal expansivity and an elasticity modulus virtually unaffected by changes in temperature. A proof mass is used as an oscillator and is attached to the center of the bottom conductive membrane (Fig. 1b).

The operating principle is based on two essential modes; drive and sense modes. Drive mode is used to bring the oscillator, i.e. the proof mass, into a rotational motion in the $x / y$ plane by applying an AC voltage with constant amplitude on drive electrodes $\left(D_{\mathrm{x} 1}, \mathrm{D}_{\mathrm{x} 2}, \mathrm{D}_{\mathrm{y} 1}\right.$ and $\left.\mathrm{D}_{\mathrm{y} 2}\right)$ and sense mode is used to readout and measure the output signals from sense electrodes $\left(\mathrm{S}_{\mathrm{x} 1}, \mathrm{~S}_{\mathrm{x} 2}, \mathrm{~S}_{\mathrm{y} 1}\right.$ and $\left.\mathrm{S}_{\mathrm{y} 2}\right)$. These drive and sense electrodes have a width of $500 \mu \mathrm{m}$ and are implemented on the top of the piezoelectric membrane. Their locations have been previously analyzed so that sense electrodes are implemented at maximum induced stress positions [24]. Attachments where sense electrodes are implemented, are designed to be tinier than those of drive electrodes in order to avoid losses and collect the totality of charges for more sensitivity.

The linear acceleration detection is accomplished based principally on exploiting the direct piezoelectric effect (Fig. 2). In fact, the applied acceleration is converted into a mechanical force given by Newton's second law ( $\mathrm{F}=\mathrm{m} . \mathrm{a})$. Depending on its direction, the seismic mass will tilt contrarily producing the maximum of deformation in the sensing electrodes location. Thanks to the direct piezoelectric effect, induced stress on the PZT membrane will be converted into an output voltage proportional to the acceleration input. On the other hand, angular rate detection employs the Coriolis effect phenomenon. Indeed, when the sensor is subjected to an 
angular motion, a deviation in the motion path of the oscillating mass arises due to the induced Coriolis force. Thus, angular velocity measurement is fundamentally related to the presence of a drive mode (primary permanent vibrating) and a second mode called sense mode. Generated Coriolis force will be orthogonal to both drive motion direction and rotation input axis. Therefore, mass vibration amplitudes in the sense mode are then proportional to the applied angular rates and coupled to the drive vibration. Since the main preeminence of the sensor is its ability to detect 6-DOF simultaneously, so the block diagram in Fig. 2 combines the two detection principles previously described.

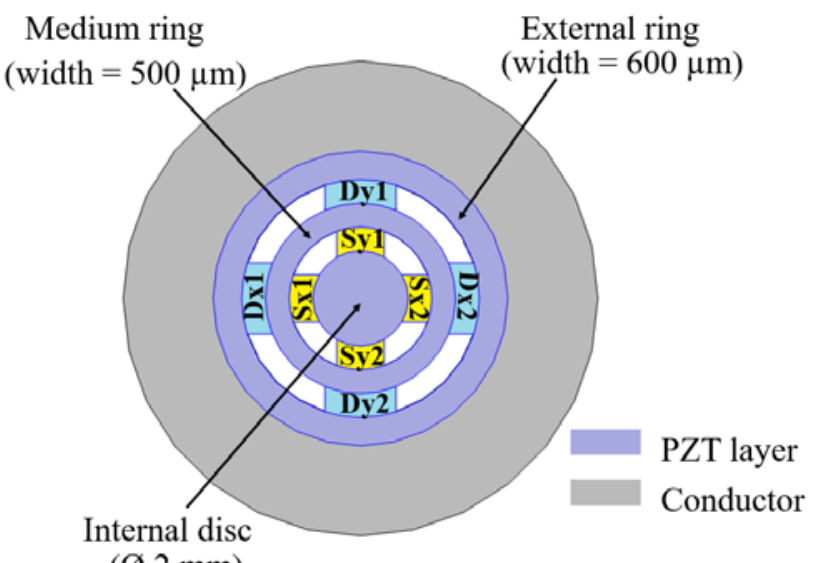
(Ø $2 \mathrm{~mm})$

(a)

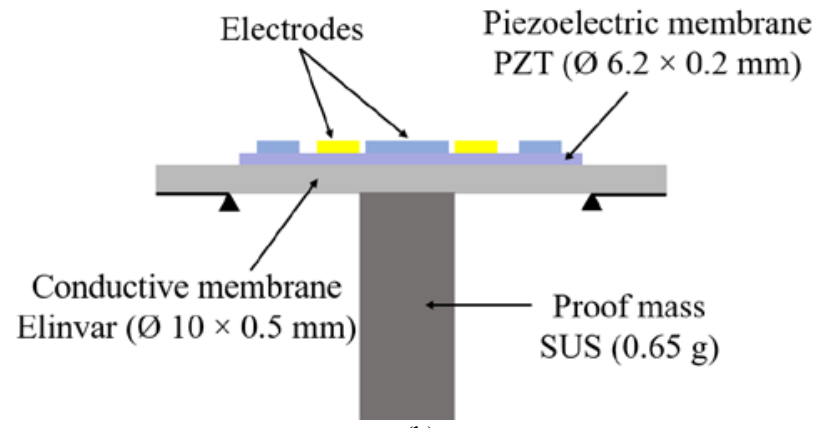

(b)

Fig. 1. Proposed Design of the New 6-Axis Inertial Sensor based on a Single Proof-Mass (a) Top view Showing Drive and Sense Electrodes Arrangement, and (b) Cross Sectional view.

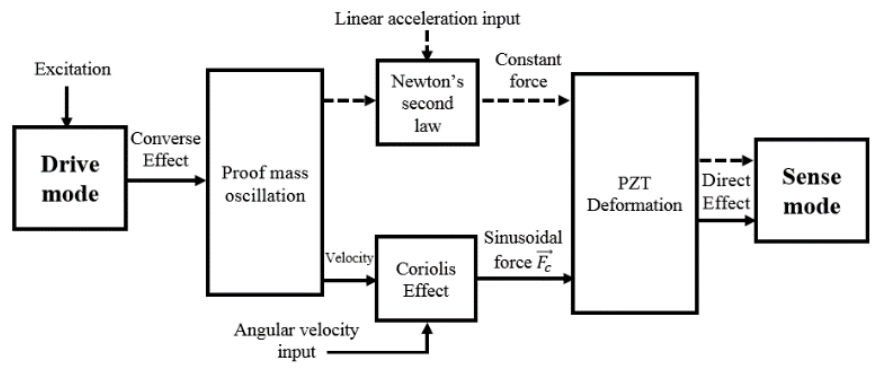

Fig. 2. Block Diagram Describing the Simultaneous Sensing of Linear and Angular Motion Principle (Acceleration Detection is based on Newton's Second Law, While Angular Rate Detection is based Coriolis Effect).

\section{ANALYTICAL ANALYSIS OF THE IMU DESIGN}

In this section we are introducing an analytical representation of the operation principle of the IMU design through the establishment of drive and sense modes equations.

Indeed, as an angular rate sensor, for each axis detection, the motion equation of the resonant sensor structure can be described using a mass-spring-damping system with two degrees of freedom as illustrated in Fig. 3. Each mode (Drive \& Sense) is then represented by a mass, m, a spring and a damper. For each mode corresponds a stiffness $\mathrm{k}$ and $\mathrm{a}$ damping factor $\eta$ (Fig. 3). An analytical description of the motion amplitude could be obtained by developing relations between the model and system variables through ordinary differential equations.

\section{A. Drive Mode Equation}

Based on the 1st fundamental principle of dynamics, projection on drive mode direction (similarly for sense mode), allows us to write:

$m \ddot{x}_{d}=F_{d}-\eta_{d} \dot{x}_{d}-k_{d} x_{d}$

where $F_{d}$ is the excitation force and $x_{d}$ is the corresponding displacement.

When considering $\omega\left(\omega_{d}^{2}=\sqrt{k_{d} / m}\right)$, and $Q\left(Q_{d}=\omega_{d} m / \eta_{\mathrm{d}}\right)$ that are, respectively, the natural frequency and the quality factor of the two modes, “(1)” becomes as follows:

$\frac{F_{d}}{m}=\ddot{x}_{d}+\frac{\omega_{d}}{Q_{d}} \dot{x}_{d}+\omega_{d}^{2} x_{d}$

By applying Laplace transform, it is possible to extract drive mode displacement given by:

$x_{d}=-j \frac{Q_{d}}{m \omega_{d}^{2}} F_{d}$

We notice that the drive vibration is in phase quadrature (90 ${ }^{\circ}$ phase shift) with the excitation force and the drive vibration amplitude is presented as follows:

$X_{d}=\frac{Q_{d}}{m \omega_{d}^{2}} F_{d}$

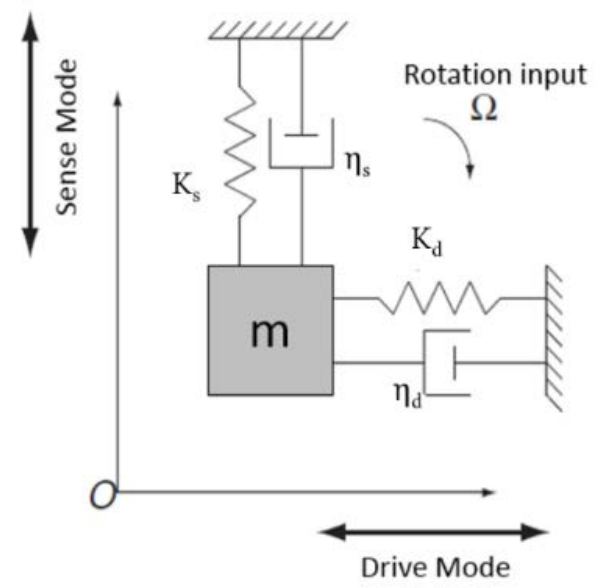

Fig. 3. Modeling of the Vibrating Sensitive Element Operation: Each Mode (Drive and Sense) is represented by a Mass-Spring-Damping System [25]. 


\section{B. Sense Mode Equation}

As explained before, under drive oscillation, sense mode is generated through the appearance of the Coriolis Effect along a perpendicular axis to both drive mode and the angular rate input direction. Thus, the equation of movement in the sense mode will be:

$m \ddot{x}_{s}=-\eta_{s} \dot{x}_{s}-k_{s} x_{s}+2 m \Omega \dot{x}_{d}$

When inserting the natural frequency and the quality factor expressions relative to sense mode vibration, the sense mode equation becomes:

$2 \Omega \dot{x}_{d}=\ddot{x}_{s}+\frac{\omega_{s}}{Q_{s}} \dot{x}_{s}+\omega_{s}^{2} x_{s}$

After passing to frequency domain, the ratio between the sense displacement $x_{s}$ and the input rotation, $\Omega$ is expressed as:

$\frac{x_{s}}{\Omega}=\frac{2 j \omega_{d} x_{d}}{\left(\omega_{s}^{2}-\omega_{d}^{2}\right)+j \frac{\left(\omega_{s} \omega_{d}\right)}{Q_{s}}}$

We consider that drive and sense resonant frequencies of the sensitive element are different $\left(\omega_{s}-\omega_{d} \neq 0\right)$ and the sense mode quality factor is important. Therefore, the expression of the sense vibration amplitude in function of the drive displacement amplitude is given by:

$x_{s}=j \frac{2 \omega_{d} \Omega}{\omega_{s}^{2}-\omega_{d}^{2}} x_{d}$

From "(8)", we can conclude that the vibration of the drive and sense mode are in quadrature. When considering that sense and drive frequencies are too close to each other but not equal, $\omega_{s}-\omega_{d}$ will be negligible compared to $\omega_{s}$. Thus, "(8)" can be concluded as follow:

$\frac{X_{s}}{\Omega}=\frac{X_{d}}{\left|\omega_{s}-\omega_{d}\right|}$

The expression exhibited in "(9)" presents the mechanical scale factor of the studied gyro-part of the 6-DOF motion sensor expressed in $(\mathrm{m} / \% / \mathrm{s})$ in terms of the drive mode vibration amplitude. Using the same theory, the dynamic response of the accelerometer is obtained in the frequency domain as:

$\frac{|X|}{F}=\frac{1}{m \sqrt{\left(\omega_{s}^{2}-\omega_{d}^{2}\right)^{2}}+4 \varepsilon^{2} \omega_{s}^{2} \omega_{d}^{2}} ; \varepsilon=\frac{\eta}{2 \sqrt{k m}}$

where $F$ is the applied force and $\varepsilon$ is the damping ratio term.

From "(9)", we can conclude that in order to maximize the scale factor of the studied motion sensor we have to maximize the drive vibration amplitude. However, this is generally limited by the maximum stress allowed by the material to avoid structural damages. In addition, we can notice that the frequency difference between drive and sense modes must be minimized. Thus, we can conclude that in order to increase the sensitivity of the sensor, the sensitive element must be dimensioned with a manner that the resonance frequencies of the two modes be very close to each other.
The illustration of analytical equations that define the sensor operation system has a great interest of understanding the theories behind the sensor operation when dealing with FEM simulations on a one hand. On another hand, this analytical approach gives a deep insight to define a set of principal parameters that influence the design. This will considerably help to optimize the sensor performances that will be exhibited in the next section.

\section{NuMERICAL INVESTIGATION OF THE 6-AXIS IMU DESIGN}

\section{A. Drive Mode Generation}

Drive mode consists in making the structure oscillate into a circular trajectory in the $x / y$ plane. This mode is chosen in such a way that the proof mass undergoes an oscillation motion in both $x$-axis and $y$-axis directions. This is achieved by applying four AC voltage signals, having a phase shift of $90 \mathrm{deg}$, to the four drive electrodes. The choice of the drive frequency is based on an Eigen frequency study that should be performed firstly through a finite element simulation to figure out the different structure normal modes. The two first inplane resonant frequencies were found to be $1481 \mathrm{~Hz}$ and 1481.1 Hz, respectively along $x$ - and $y$-axes as shown in Figs. 4a and $4 \mathrm{~b}$. Indeed, symmetrical flexure is obviously present along both $x$ - and $y$-axis which produces the same modal frequency of the design. The next modes, shown in Fig. 4c and $4 \mathrm{~d}$, are non-axisymmetric. The next out-of-plane mode along $\mathrm{z}$-axis, was found to be around $18 \mathrm{kHz}$ (Fig. 4e). Mode 1 will be the one used for successfully completing the proposed configuration.

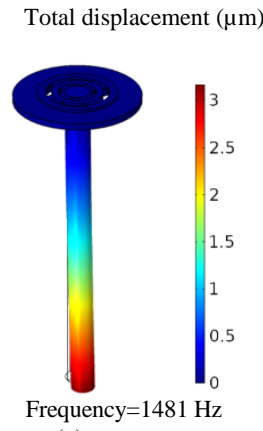

(a)

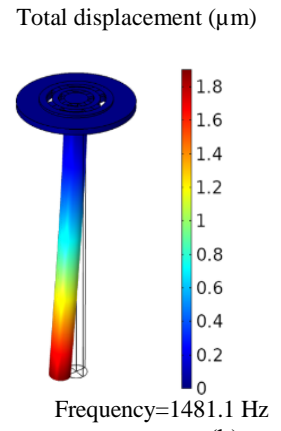

(b)

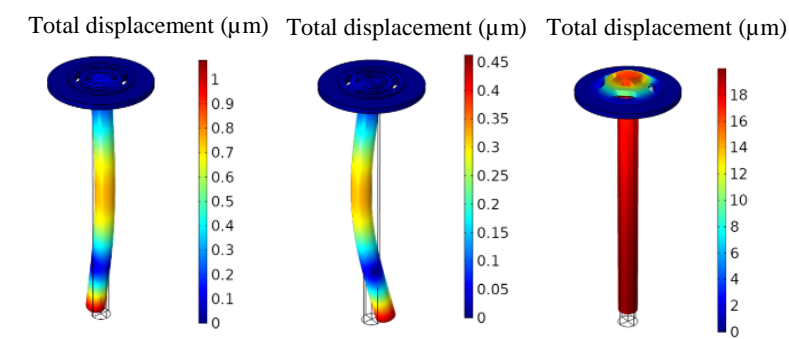

(c)
Frequency $=10187 \mathrm{~Hz}$ (d) (e)
Fig. 4. Simulated (a) and (b) First in-Plane Modes of Vibration along $x$-Axis and $y$-Axis, respectively; (c) and (d) Non-Axisymmetric Modes and (e) Out-of Plane mode of Vibration along z-axis. 


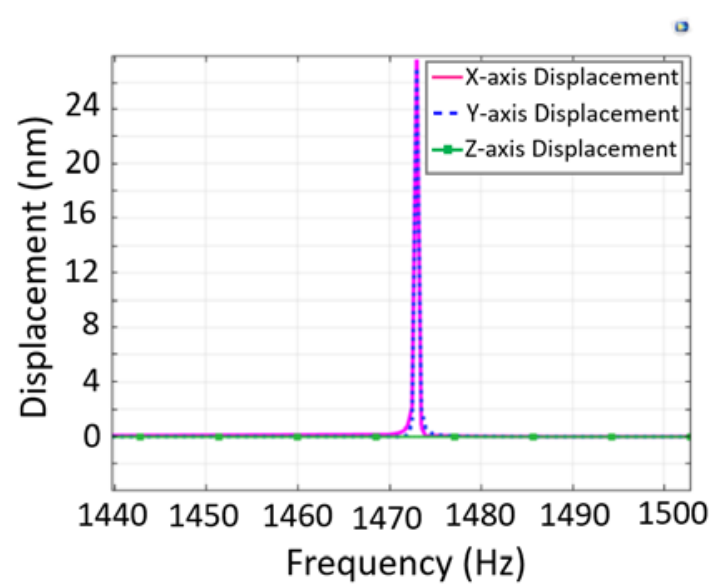

Fig. 5. $\mathrm{x}-$, $\mathrm{y}-$ and $\mathrm{z}$-Axis Proof Mass Displacement in response to a Frequency Sweep.

Fig. 5 shows the displacement magnitude of the lower median tip of the seismic mass as a function of a range of excitation frequency, around the resonance, using FEM simulations (under a drive amplitude voltage of $2 \mathrm{mV}$ ). In the simulated range, $z$-axis displacement is found with a very low magnitude that could be neglected, which confirm the proof mass oscillation in the $x / y$ plane. It is worth noting that the chosen frequency of drive mode faithfully corresponds to this pic value that is near to the first axisymmetric mode of both $\mathrm{x}$ and $y$-axis which leads to a maximum proof mass displacement while using the minimum supply voltage.

\section{B. Mechanism of 6-DOF Detection}

This section is to illustrate FEM simulation results of the studied IMU design. An investigation of signals output nature relative to linear and angular motion detection is evaluated. Also, the sensor footprint and its operating performances are extracted to be compared with other designs from literature.

\section{Linear Acceleration}

The analysis of voltage outputs relative to acceleration inputs is performed using a temporal FEM study [26]. The latter should be realized in practical conditions, since the structure should be permanently biased with drive voltage signals in order to be able to measure the angular velocity once applied. Upon an $\mathrm{x}$-axis acceleration input, the electrical potential generated in two aligned electrodes will be opposite since one will undergo a compression and the other a dilatation. Hence, the harvested voltage is maximized by differentiating between potentials in Sx1 and Sx2 electrodes. FEM results show that the output signals, when drive mode is ongoing, give a DC offset shift proportional to the experienced acceleration superimposed to the AC signal corresponding to the drive mode reference signal (Fig. 6a). Thus, a conditioning circuitry is needed to eliminate the permanent AC signal generated by the drive mode. Processed signals offset relative to $\mathrm{x}$-axis acceleration input is depicted in Fig. 6b showing a sensitivity of $364 \mathrm{mV} / \mathrm{g}$. Similarly, for y-axis acceleration, output signals are obtained by using a differential potential between Sy1 and Sy2. Since the design represents a radial (or rotational) symmetry around $\mathrm{z}$-axis, y-axis acceleration generates the same output voltages values as found with the application of $\mathrm{x}$-axis acceleration inputs. For an acceleration along z-axis, the whole structure will undergo a vertical displacement, therefore the voltage generated on the four electrodes will have the same value and vary in the same direction (+ or - ). Hence, output signal is collected from the sum of the four sense electrodes. Fig. 7b exhibits numerical simulations in response to z-axis acceleration input with the presentation of the resulted cross-axis signals showing a sensitivity of $65.5 \mathrm{mV} / \mathrm{g}$.

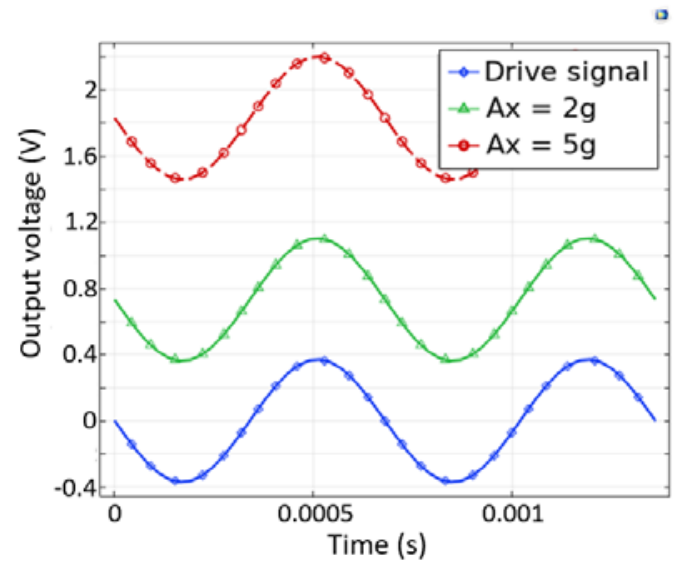

(a)

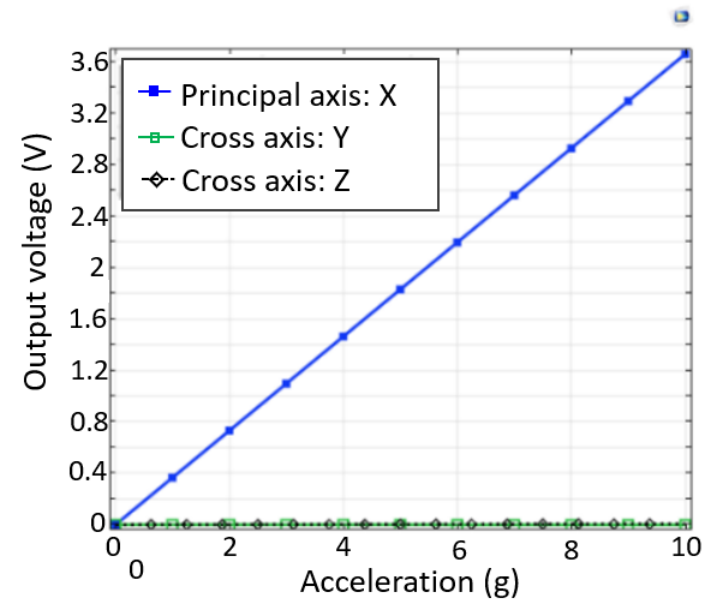

(b)

Fig. 6. FEM Simulation Result of Output Voltage $\left(\mathrm{S}_{\mathrm{x} 1}-\mathrm{S}_{\mathrm{x} 2}\right)$ in response to $\mathrm{x}$ Axis Linear Acceleration Input under (a) Drive Mode and (b) Static Study.

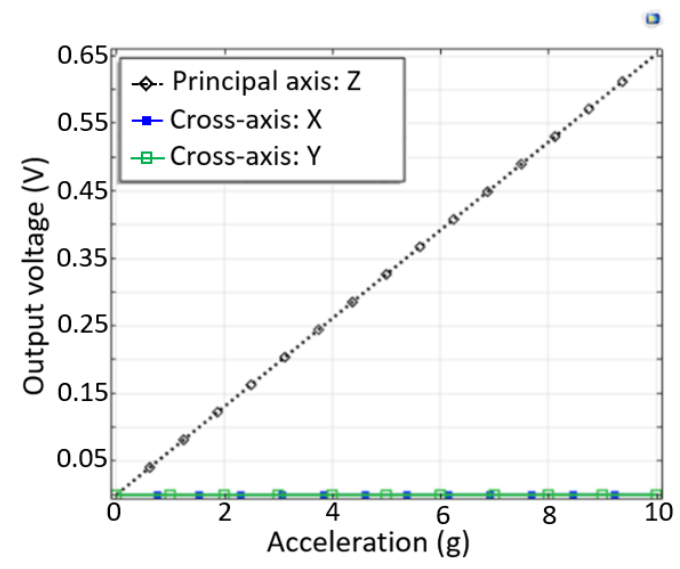

Fig. 7. FEM Simulation Result of Output Voltage in response to $y$ - and zAxis Linear Acceleration Input showing a Good Cross-Axis Sensitivity. 


\section{Linear Cross-Axis Sensitivity}

Cross-axis sensitivity in accelerometers is considered as an essential property especially for high performance applications. It is defined as:

Cross_Sens $=\frac{\sqrt{S_{c 1}^{2}+S_{c 2}^{2}}}{S} \times 100 \%$

where $S$ is the measured sensitivity in the considered direction and $S_{c 1}$ and $S_{c 2}$ are the measured sensitivities in the two other cross-axis directions.

The proposed novel design presents an excellent characteristic in term of cross-axis sensitivity; the structure gives $0.05 \%$ as cross-axis sensitivity for transversal axes and $0.02 \%$ for $\mathrm{z}$-axis. Thus, it is efficiently ensured that the fact of detecting the acceleration motion in a principal axis will not affect the other axes. This crucial property is obtained thanks to the highly symmetrical structure of the model that can significantly restrain the cross-axis sensitivity without affecting the sensor sensitivity making the proposed design a highly performance inertial sensor.

\section{E. Angular Rate}

When the sensor is subjected to an angular motion, $\Omega$, a Coriolis force, $F_{c}$, will be generated as given by:

$\overrightarrow{F_{c}}=2 m \vec{v} \times \vec{\Omega}$

where $m$ is the proof-mass mass, and $\mathrm{v}$ is the applied velocity component of the oscillator (displacement derivative included as well in $x / y$-plane due to the drive mode). When a longitudinal angular rate input acts $\left(\Omega_{x}\right.$ or $\left.\Omega_{y}\right)$, two components of Coriolis force are generated since $z$-axis is perpendicular to the $x / y$ oscillation plane. So, both $x$-and $y$ axis angular velocity will be detected by the production of Coriolis force along z-axis, as:

$F_{c z}=-2 m v_{y} \Omega_{x}$

$F_{c z}=2 m v_{x} \Omega_{y}$

Thus, in presence of a longitudinal angular rotation, the planar circular oscillation trajectory of the proof mass will be perturbed instantly due to the sinusoidal Coriolis force along $\mathrm{z}$-axis. In fact, an oscillation along $\mathrm{z}$-axis will be added, and therefore, the oscillation plane will have a well-defined inclination with respect to the initial horizontal x/y-plane (Fig 8). Hence, this inclination induces a change in the amplitude of the generated AC signal compared to that from the drive mode. In response to $\Omega_{\mathrm{x}}$, the difference in amplitudes between drive and sense signals recovered from the sense electrodes placed in y-direction $\left(\mathrm{S}_{\mathrm{y} 1}-\mathrm{S}_{\mathrm{y} 2}\right)$ will be significantly higher (Fig. 9a) than the one recovered from electrodes along $\mathrm{x}$-axis $\left(\mathrm{S}_{\mathrm{x} 1}-\mathrm{S}_{\mathrm{x} 2}\right)$ as depicted in Fig. 9b. This means that $\Omega_{\mathrm{x}}$ readout will occur only from $\mathrm{S}_{\mathrm{y}}$ electrodes, and inversely for $\Omega_{\mathrm{y}}$. This fact leads to good cross-axis sensitivities in both $\mathrm{x}$ - and $\mathrm{y}$-axis. In practice, total voltage is measured from the summation of potential difference between $\mathrm{x}$-sense electrodes $\left(\mathrm{S}_{\mathrm{x} 1}-\mathrm{S}_{\mathrm{x} 2}\right)$ and $\mathrm{y}$-sense electrodes $\left(\mathrm{S}_{\mathrm{y} 1}-\mathrm{S}_{\mathrm{y} 2}\right)$. Besides, for $\mathrm{x}$-axis angular input, we must pay attention to the presence of $y$-axis component of the Coriolis force. This latter will be produced due to the presence of z-axis velocity (even with an extremely low value) that will also be combined with $\mathrm{x}$-axis angular input $\left(F_{c y}=2 \mathrm{~m}\right.$ $v_{z} \Omega_{x}$ ). Consequently, the seismic mass will then undergo a significant additional oscillation stretched in the y-direction that will be manifested in an elliptical curve oscillation instead of a perfect circular trajectory.

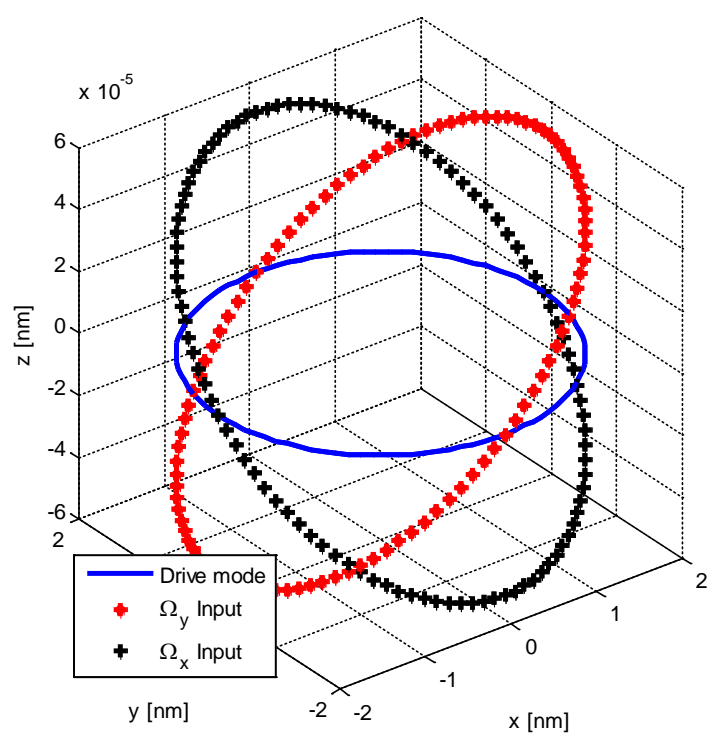

Fig. 8. Illustration of the Proof Mass Trajectory under Drive mode and in response to $\mathrm{x}$ - and $\mathrm{y}$-axis Angular Rate Input.

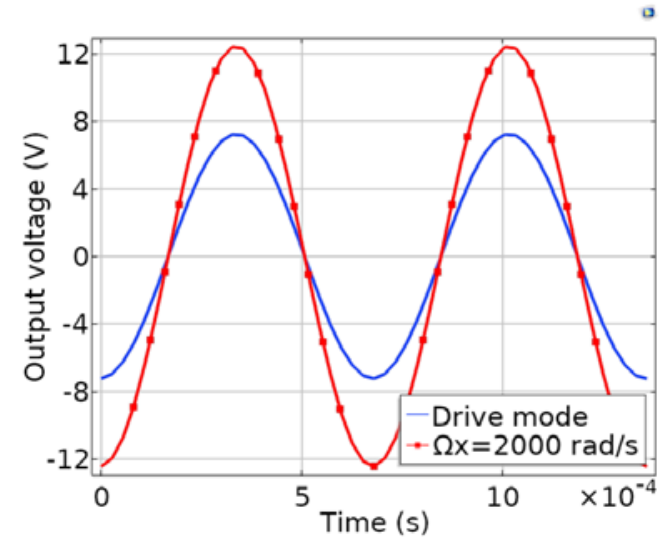

(a)

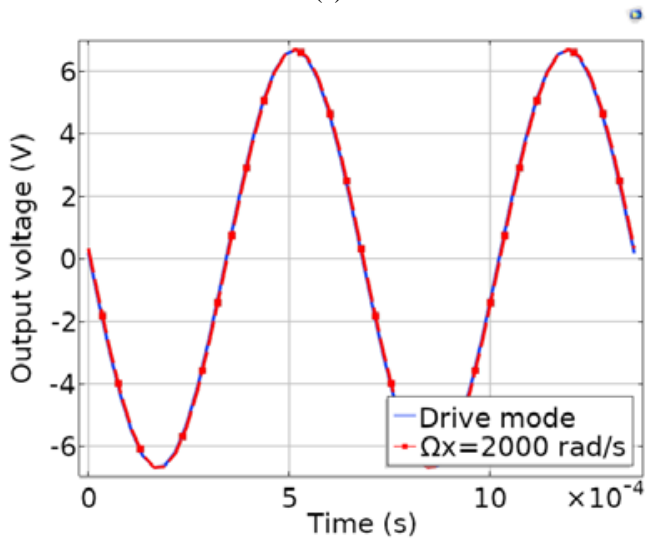

(b)

Fig. 9. Output Voltages Obtained in response to an $\mathrm{x}$-axis Angular Rate from (a) $\mathrm{S}_{\mathrm{y} 1}-\mathrm{S}_{\mathrm{y} 2}$, and (b) $\mathrm{S}_{\mathrm{x} 1}-\mathrm{S}_{\mathrm{x} 2}$. 
When a $z$-axis angular rate input acts on the sensor, two components of Coriolis force are generated, and the oscillating sensor will be disturbed by a supplementary vibration in the $x$ or $y$-axis directions due to the generation of Coriolis effect exhibited by:

$F_{c x}=2 m v_{y} \Omega_{z}$

$F_{c y}=2 m v_{x} \Omega_{z}$

Using FEM simulation results, we can extract the sensor response under $\Omega_{\mathrm{z}}$ input through the evaluation of the effect of Coriolis force produced in both $x$ - and $y$-directions.

Consequently, the diameter of the circular oscillation trajectory of the seismic mass will either increase or decrease (Fig. 10). Therefore, the amplitude of the sinusoidal component of all sense electrodes will either increase or decrease, depending on the direction of the angular rotation.

In Fig. 11 output voltages versus time are extracted from the potential difference between Sx and Sy electrodes showing an equal amplitude value. Minimum and maximum angular rate values that the proposed sensor can linearly read in transversal and longitudinal axes are respectively $\pm 2000 \mathrm{rad} / \mathrm{s}$ and $\pm 5 \mathrm{rad} / \mathrm{s}$. Angular rate sensitivities are numerically evaluated in all three-dimensional axes and are listed in Table I.

\section{F. Discussion, Design Footprint and Comparison with other Designs from Literature}

Detailed performances and characteristics of the novel IMU design and other piezoelectric inertial sensor designs from literature are illustrated in Table I. In term of linear acceleration detection, results reveal that the new proposed design allows a higher sensitivity compared to other accelerometer designs reported in $[27,28]$.

For angular rate sensing, our proposed structure gives considerable sensitivity value especially when compared with Chang et al. [29]. Besides, among the reported works, the novel IMU design exhibits the highest angular rate sensitivity along $z$-axis.

Main specifications of the developed IMU design are summarized in Table II as sensor footprint.
In our proposed design, for both linear and angular inputs, voltage output signals will be collected from the four sense electrodes. Changes in every electrode for each input case (linear and angular motions) are illustrated in Table III.

This illustration will help to design the circuitry of separation of the 6-DOF from the IMU and ensure the concurrent detection. It is worth noting that the drive mode will induce an initial voltage on sense electrodes with an amplitude ' $A$ '. ' $\varphi$ ' denotes the phase of the signal and 'ofs' is the offset value of the signal.

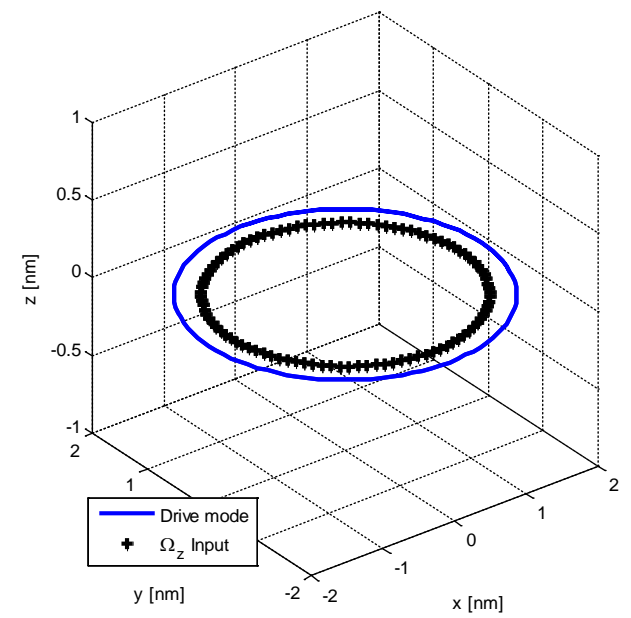

Fig. 10. Illustration of the Proof Mass Trajectory under Drive Mode and in response to z-axis Angular Rate Input.

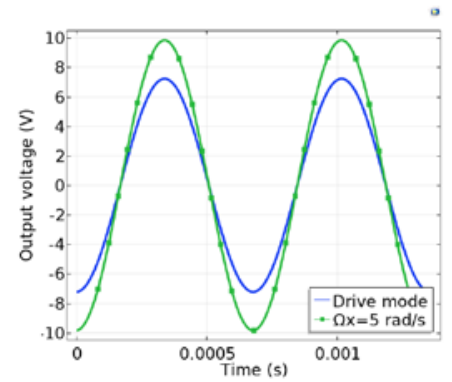

(a)

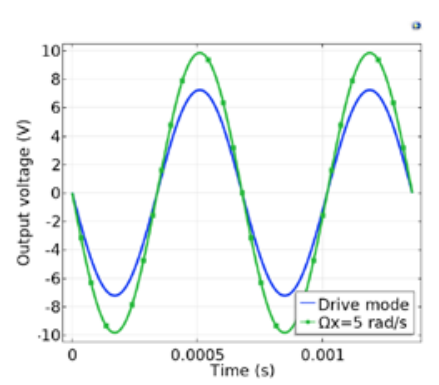

(b)
Fig. 11. Output Voltage Measured in response to z-axis Angular Rate Input from (a) $S_{x 1}-S_{x 2}$ and (b) $S_{y 1}-S_{y 2}$.

TABle I. Characteristics ANd Performances in Term of AcCeleration ANd ANGUlar Rate Detection by the Proposed Design AND other PIEZOELECTRIC DESIGNS REPORTED FROM LITERATURE

\begin{tabular}{|c|c|c|c|c|c|c|}
\hline \multirow{3}{*}{ Design } & \multirow{3}{*}{ Dimensions scale } & \multirow{3}{*}{$\begin{array}{l}\text { Piezo-electric } \\
\text { Material }\end{array}$} & \multicolumn{4}{|l|}{ Sensitivity } \\
\hline & & & \multicolumn{2}{|l|}{ Acceleration } & \multicolumn{2}{|l|}{ Angular rate } \\
\hline & & & $A_{x} \& A_{y}$ & $\mathrm{~A}_{\mathrm{z}}$ & $\Omega_{\mathrm{x}} \& \Omega_{\mathrm{y}}$ & $\Omega_{\mathrm{z}}$ \\
\hline Novel IMU & Mesoscale & PZT & $\begin{array}{l}364 \\
\mathrm{mV} / \mathrm{g}\end{array}$ & $\begin{array}{l}65.5 \\
\mathrm{mV} / \mathrm{g}\end{array}$ & $\begin{array}{l}2.59 \\
\mathrm{mV} / \mathrm{rad} / / \mathrm{s}\end{array}$ & $\begin{array}{l}522 \\
\mathrm{mV} / \mathrm{rad} / \mathrm{s}\end{array}$ \\
\hline Wang et al. [27] & MEMS & $\mathrm{ZnO}$ & \multicolumn{2}{|l|}{$16.3 \mathrm{mV} / \mathrm{g}$} & & \\
\hline Shen et al. [28] & MEMS & PLZT & \multicolumn{2}{|l|}{$31.15 \mathrm{mV} / \mathrm{g}$} & \multirow{2}{*}{\multicolumn{2}{|c|}{$1.82 \mu \mathrm{V} / \mathrm{deg} / \mathrm{s}$}} \\
\hline Chang et al. [29] & Mesoscale & $\mathrm{PZT}$ & & & & \\
\hline Fujii et al. [30] & MEMS & $\mathrm{PZT}$ & \multicolumn{2}{|l|}{$x^{2}$} & \multicolumn{2}{|l|}{$25 \mathrm{mV} / \mathrm{deg} / \mathrm{s}$} \\
\hline Parent et al. [31] & Mesoscale & PZT & \multirow{2}{*}{\multicolumn{2}{|c|}{$300 \mathrm{mV} / \mathrm{g}$}} & \multicolumn{2}{|l|}{$3 \mathrm{mV} / \mathrm{deg} / \mathrm{s}$} \\
\hline Okada et al. [10] & Mesoscale & $\mathrm{PZT}$ & & & $2.5 \mathrm{mV} / \mathrm{deg} / \mathrm{sec}$ & \\
\hline LV et al. [32] & Mesoscale & Quartz & $0.695 \mathrm{pC} / \mathrm{m} / \mathrm{s}^{2}$ & $0.423 \mathrm{pC} / \mathrm{m} / \mathrm{s}^{2}$ & $0.028 \mathrm{pC} / \mathrm{rad} / \mathrm{s}^{2}$ & $0.023 \mathrm{pC} / \mathrm{rad} / \mathrm{s}^{2}$ \\
\hline
\end{tabular}


TABLE II. NOVEL IMU DESIGN FOOTPRINT

\begin{tabular}{|l|}
\hline Concurrent 6-DOF detection Linear acceleration and angular rate sensing \\
\hline High sensitivity Up to $522 \mathrm{mV} / \mathrm{rad} / \mathrm{s}$ \\
\hline Very low cross-axis sensitivity Up to $0.02 \%$ \\
\hline IMU based on a single-core design instead of two sensors \\
\hline
\end{tabular}

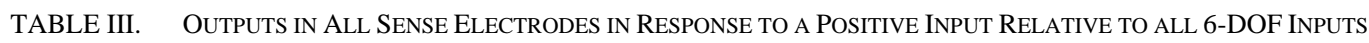

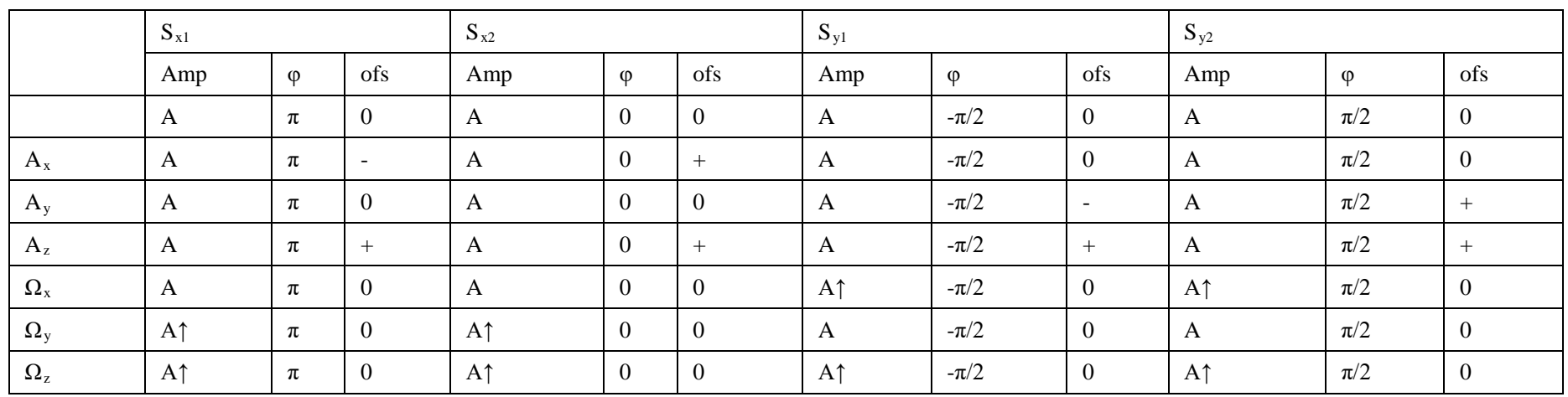

\section{6-DOF SEPARATION CIRCUITRY}

In the presence of acceleration or angular velocity components, initial voltage from drive voltage will be superimposed to the sensing signals on the same sense electrodes. The main function of the developed IMU sensor is to deal with linear and angular motion concurrently, using a single resonant structure. Hence, the challenge consists mainly in the differentiation of the linear and angular measured outputs from only four electrodes in order to be able to identify the nature and magnitude of the applied input. The used processing signal bloc diagram, operating on sensing electrodes is exhibited in Fig. 12, and signal evolution on the used sense electrodes combinations for 6-DOF inputs, are illustrated in Table IV. Basically, any accelerations will change the offset of the signals in the electrodes, while the angular accelerations will change the drive mode amplitude of the sinusoidal signals.

The separation circuitry proposed for linear acceleration detection from the four electrodes is given in Fig. 13. The principle is based on the detection of the signal offset, proportional to the acceleration input, which is the key data to differentiate between signals coming from angular or linear inputs. For $x$-and $y$-axis linear acceleration, a differential operational is used to generate $S_{x}\left(=S_{x 1}-S_{x 2}\right)$ and $S_{y}\left(=S_{y 1}\right.$ $S_{\mathrm{y} 2}$ ) based on signals collected from the four electrodes. For $\mathrm{z}-$ axis acceleration, a summing amplifier is used to generate $\mathrm{S}_{\mathrm{x} 1}+\mathrm{S}_{\mathrm{x} 2}+\mathrm{S}_{\mathrm{y} 1}+\mathrm{S}_{\mathrm{y} 2}$. The acceleration detection circuit has the role to eliminate the AC drive signal and keep only the offset voltage. Both Signals from amplifiers are passed initially through a low pass filter with a cut-off frequency much lower than the sinusoidal drive frequency (i.e. $1471.5 \mathrm{~Hz}$ ). The low pass filter is used to keep only the offset value of the signal. Lastly, the detected signal is passed through a voltage follower for impedance matching and for preventing loading effect [27]. The overall transfer function of the $x$-and $y$-axis acceleration detection circuit is given by:

$$
H(j w)=\left(\frac{R 2}{R 1}\right)\left(\frac{1}{1+j R 3 C 1 \omega}\right)
$$

The output signal will be divided to be integrated into the acceleration detection circuit and the angular rate detection circuit. The angular rate detection circuit is divided into two stages. In the first stage, shown in Fig. 13, a coupling capacitor is used to eliminate the DC offset from $S_{x} / S_{y}$ signals. Next, a differential amplifier serves to remove the permanent AC signal, belonging to the drive mode, from the output signal provided by $\mathrm{S}_{\mathrm{x} 1} / \mathrm{S}_{\mathrm{y} 1}$. Then, a peak detection circuit is used to store the positive peak amplitude of the difference signal. The peak detector uses an operational amplifier connected in series with diode and a capacitor (Fig. 13). It is worth remembering that the initial voltage depends only on the drive input signal amplitude, so a calibration step is needed at the outset. Hence the output signal represents a DC voltage proportional to the angular rate input recorded in $\mathrm{S}_{\mathrm{x}} / \mathrm{S}_{\mathrm{y}}$ electrode. The proposed circuit depicted in Fig. 13 is useful for differentiating an acceleration data from an angular one as illustrated in Fig. 14. To identify which angular rate input (i.e. $\Omega_{\mathrm{x}}, \Omega_{\mathrm{y}}$ or $\Omega_{\mathrm{z}}$ ) represents this information, a second stage needs to be added.

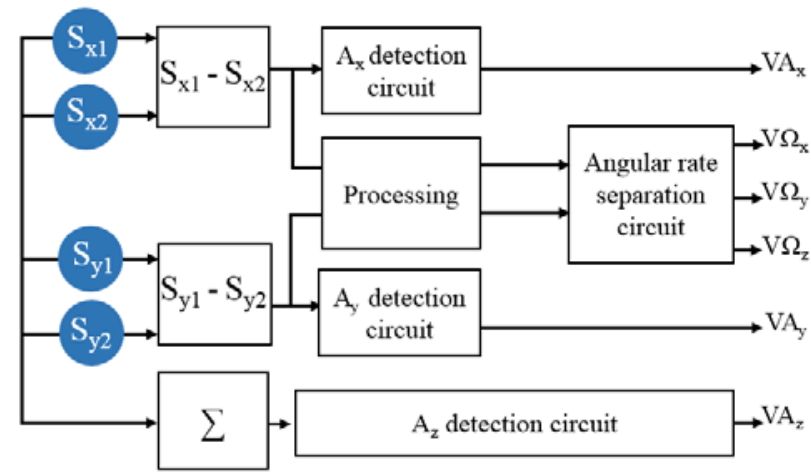

Fig. 12. Bloc Diagram of Signal Separation Circuit between the 6-DOF Inputs. 
TABLE IV. OUTPUTS IN ALL SENSE ELECTRODES IN RESPONSE TO A POSITIVE INPUT RELATIVE TO ALL 6-DOF INPUTS.

\begin{tabular}{|c|c|c|c|c|c|c|c|c|c|}
\hline \multirow{3}{*}{$\mathrm{A}_{\mathrm{x}}$} & \multicolumn{3}{|c|}{$\mathrm{S}_{\mathrm{x} 1}-\mathrm{S}_{\mathrm{x} 2}$} & \multicolumn{3}{|c|}{$\mathrm{S}_{\mathrm{y} 1}-\mathrm{S}_{\mathrm{y} 2}$} & \multicolumn{3}{|c|}{$\mathrm{S}_{\mathrm{x} 1}+\mathrm{S}_{\mathrm{x} 2}+\mathrm{S}_{\mathrm{x} 1}+\mathrm{S}_{\mathrm{x} 2}$} \\
\hline & Amp & $\varphi$ & ofs & Amp & $\varphi$ & offset & Amp & $\varphi$ & ofs \\
\hline & B & $\pi$ & $\lambda$ & \multicolumn{3}{|c|}{ Zero signal } & \multicolumn{3}{|c|}{ Zero signal } \\
\hline $\mathrm{A}_{\mathrm{y}}$ & \multicolumn{3}{|c|}{ Zero signal } & B & $-\pi / 2$ & $\nearrow$ & \multicolumn{3}{|c|}{ Zero signal } \\
\hline$A_{z}$ & \multicolumn{3}{|c|}{ Zero signal } & \multicolumn{3}{|c|}{ Zero signal } & $\mathrm{C}$ & $\pi$ & $\lambda$ \\
\hline$\Omega_{\mathrm{x}}$ & $\mathrm{B}$ & $\pi$ & 0 & $\mathrm{~B} \uparrow$ & $-\pi / 2$ & 0 & $\mathrm{C} \uparrow$ & $\pi$ & 0 \\
\hline$\Omega_{\mathrm{y}}$ & $\mathrm{B} \uparrow$ & $\pi$ & 0 & $\mathrm{~B}$ & $-\pi / 2$ & 0 & $\mathrm{C} \uparrow$ & $\pi$ & 0 \\
\hline$\Omega_{\mathrm{z}}$ & $\mathrm{B} \uparrow$ & $\pi$ & 0 & $\mathrm{~B} \uparrow$ & $-\pi / 2$ & 0 & \multicolumn{3}{|c|}{ Zero signal } \\
\hline
\end{tabular}

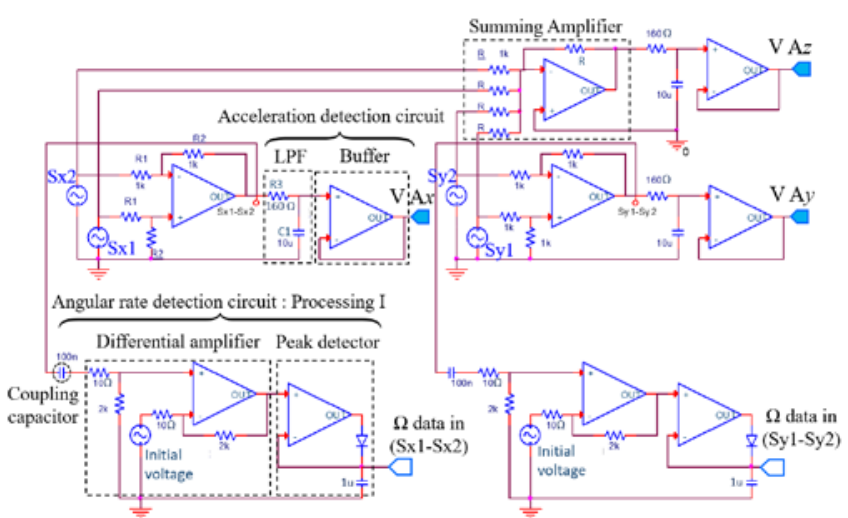

Fig. 13. Diagram of the Simultaneous Detection Circuit of Linear Acceleration and Angular Velocity Signals.

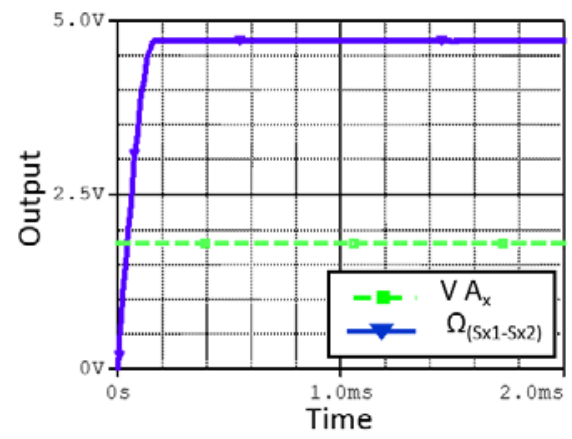

Fig. 14. Output Signals Collected from $\mathrm{VA}_{\mathrm{x}}$ Output upon an $\mathrm{x}$-axis Linear Acceleration of $5 \mathrm{~g}$, and from $\Omega\left(\mathrm{S}_{\mathrm{x} 1}-\mathrm{S}_{\mathrm{x} 2}\right)$ Output upon an Angular Velocity of $2000 \mathrm{rad} / \mathrm{s}$.

Outputs relative to $x$-, $y$-, and $z$-axis angular rates are recognized based on the data collected simultaneously from $\Omega_{(\mathrm{Sx} 1-\mathrm{Sx2})}$ and $\Omega_{(\mathrm{Sy1}-\mathrm{Sy} 2)}$ outputs. Indeed, as depicted in Table IV,$\Omega_{\mathrm{z}}$ is detected only if the same data are collected in both outputs $\Omega_{\text {(Sx1-Sx2) }}$ and $\Omega_{\text {(Sy1-Sy2) }}$. Whereas, $\Omega_{\mathrm{x}}$ is detected when considering only a change in $\Omega_{\text {(Sy1-Sy2) }}$ signal and the change in only $\Omega_{(\mathrm{Sx1}-\mathrm{Sx} 2)}$ is devoted to $\Omega_{\mathrm{y}}$ detection. Thus, the angular rate dissociation circuit is designed as follow: $\Omega_{(\mathrm{Sx1}-\mathrm{Sx} 2)}$ and $\Omega_{\text {(Sy1-Sy2) }}$ data are compared in parallel with two op-amp voltage comparators $\left(\Omega_{\text {(Sx1-Sx2) }}\right.$ is on the positive input of the first comparator, and $\Omega_{\text {(Sy1-Sy2) }}$ is on the positive input of the second one). The two outputs of the comparators are then passed through a logic NOR gate. The appropriate signal output is realized by two analog demultiplexers whose outputs are controlled by the NOR gate. At the moment of same data are collected from $\Omega_{\text {(Sx1-Sx2) }}$ and $\Omega_{\text {(Sy1-Sy2) }}$ outputs, the input of each demultiplexer is transmitted to the 'Y1' pin. Then, by using a non-inverting summing amplifier followed by a buffer, the output voltage corresponding to $\Omega_{\mathrm{z}}$ is faithfully gathered from the two demultiplexers. In the opposite case $\left(\Omega_{(\mathrm{Sx1}-\mathrm{S} \times 2)}\right.$ and $\Omega_{(\text {Sy1-Sy2) }}$ are different), $\Omega_{(\text {Sx1-Sx2) }}$ and $\Omega_{(\text {Sy1-Sy2) }}$ data are transmitted to 'Y0' pin of each demultiplexer, and the signal will refer to either $\Omega_{\mathrm{x}}$ or $\Omega_{\mathrm{y}}$ angular rate depending on which signal is non-zero as explained before. The block diagram and simulation results of the proposed circuit are respectively depicted in Fig. 15 and Fig. 16.

The used circuit for angular rate dissociation represents a very efficient method for signal separation since no leakage of angular rate readout occurs in all three axes. This fact makes the proposed 6-DOF IMU design a highly performed sensor showing practically no cross-axis in all 6-axis. Other particular interest is that under no circumstances, angular outputs disturb or affect acceleration ones and vice versa. This fact is ensured thanks to the use of the specific electronic circuitry. Further works can shed light on the study of a selfexciting drive mode circuit in order to ensure perfect oscillation conditions.

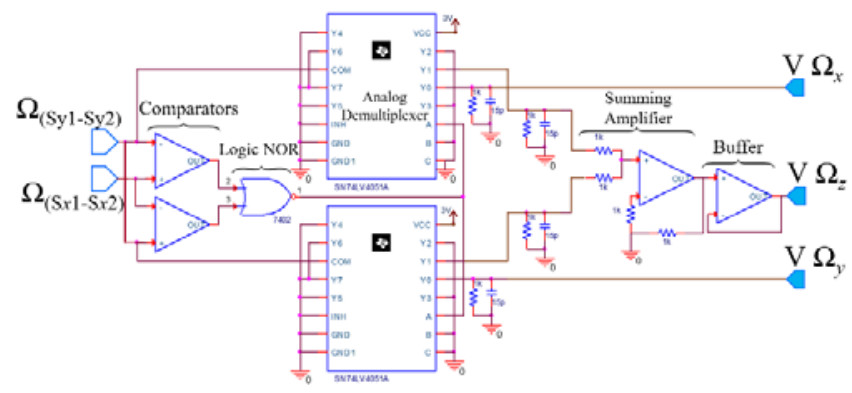

Fig. 15. Bloc Diagram of Separation Circuit between x-, y-, and z-axis Angular Rate Input.

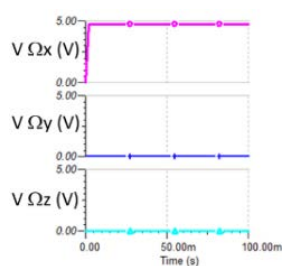

(a)

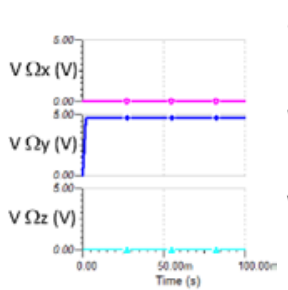

(b)

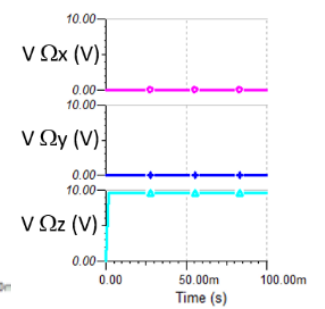

(c)
Fig. 16. Output Simulation from (a) $\Omega_{\mathrm{x}}$, (b) $\Omega_{\mathrm{y}}$, and (c) $\Omega_{\mathrm{z}}$ Inputs using the Proposed Circuitry. 


\section{CONCLUSION}

This paper reports the operation principles of 6-DOF detection using a new piezoelectric IMU design based on a single proof mass oscillator. The major asset is to combine acceleration and angular rate sensing into a single-core device. The sensor performances and characteristics were investigated from finite elements simulations. Thanks to its highly symmetrical structure, the novel IMU design exhibited very low cross-axis sensitivity values and in term of sensitivity, it has shown great performances when compared with other piezoelectric designs. Linear acceleration sensitivity was found to be $364 \mathrm{mV} / \mathrm{g}$ for both $x$-and $y$-axis, and $65.5 \mathrm{mV} / \mathrm{g}$ for the $z$-axis constituent. In term of angular rate detection, the sensitivity was found to be $2.59 \mathrm{mV} / \mathrm{rad} / \mathrm{s}$ for both $x$-and $y$ axis, and $522 \mathrm{mV} / \mathrm{rad} / \mathrm{s}$ for the $z$-axis. A complete conditioning circuit detection was efficiently designed for the separation of linear and angular readouts using a minimal number of electrodes (only four electrodes) and the concurrent detection of 6-DOF is achieved.

\section{REFERENCES}

[1] V. Saahar and R. Durai, "Designing MEMS Based Tuning Fork Gyroscope For Navigation Purpose," International conference on Communication and Signal Processing, pp. 1102-1107, April, 2013.

[2] B. Mashadi and M. Gowdini, "Vehicle Dynamics Control by Using an Active Gyroscopic Device,” Journal of Dynamic Systems Measurement and Control, vol. 137, pp. 1-12, 2015.

[3] C. Acar, A. Schofield, A.R. Trusov, L.E Costlow and A.M. Shkel, "Environmentally robust MEMS vibratory gyroscopes for automotive applications,” IEEE Sensors Journal, vol. 9, pp. 1895-1906, 2009.

[4] B. Munoz-Barron, J. R. Rivera-Guillen, R. A. Osornio-Rios and R. J. Romero-Troncoso; "Sensor Fusion for Joint Kinematic Estimation in Serial Robots Using Encoder, Accelerometer and Gyroscope,” Journal of Intelligent \& Robotic Systems, vol. 78, pp. 529-540, 2015.

[5] Z. You, "Space Microsystems and Micro/nano Satellites," Micro and Nano Technologies Book Series, pp. 233-293, 2018.

[6] A.S. Kundu, O. Mazumder, P.K. Lenka and S. Bhaumik, "Hand Gesture Recognition Based Omnidirectional Wheelchair Control Using IMU and EMG Sensors,” Journal of Intelligent and Robotic Systems, vol. 91, pp. 529-541, 2018.

[7] E. Bostanci, B. Bostanci, N. Kanwal and A. F. Clark, "Sensor fusion of camera, GPS and IMU using fuzzy adaptive multiple motion models," Soft Computing, vol. 22, pp. 2619-2632, 2018.

[8] D.A. Gura, G.G. Shevchenko, L.F. Kirilchik, D.V. Petrenkov and T. A. Gura, "Application of inertial measuring unit in air navigation for ALS and DAP," Journal of Fundamental and Applied Sciences, vol.9, pp.732-741, 2017.

[9] N. Ahmad, R. A. R. Ghazilla and N. M. Khairi, "Reviews on Various Inertial Measurement Unit (IMU) Sensor Applications,” International Journal of Signal Processing Systems, vol. 1, pp. 256-262, 2013.

[10] K. Okada, T. Kakutani, H. Itano, Y. Matsu and S. Sugiyama, "Development of 6-axis Motion Sensors Using Piezoelectric Elements," in Proceedings of the 21st Sensor Symposium on Sensors, Micromachines and Applied Systems, October 14 - 15, Kyoto, Japan, 2004.

[11] A. Parent, O.L. Traon, S. Masson and B.L. Foulgoc, "A Coriolis Vibrating Gyro Made of a Strong Piezoelectric Material," In Proceedings of IEEE Sensors, pp. 876-879, Atlanta, GA, USA, October 2007.

[12] F. Mailly, A. Martinez, A. Giani, F. P. Delannoy and A. Boyer, "Design of a micromachined thermal accelerometer: thermal simulation and experimental results,”Microelectronics Journal,vol.34,pp.275-280,2003.

[13] S. Habibi, S. J. Cooper, J-M. Stauffer and B. Dutoit, "Gun hard inertial measurement unit based on MEMS capacitive accelerometer and rate sensor," in IEEE/ION Position, Location and Navigation Symposium, pp. 232-237, May 2008.
[14] A. Partridge, J.K. Reynolds, B.W. Chui, E. M. Chow, A.M. Fitzgerald, L. Zhang, N.I. Maluf and T.W. Kenny, "A High-Performance Planar Piezoresistive Accelerometer," Journal of Microelectromechanical Systems, vol. 9, pp. 58-66, March 2000.

[15] J. Wagner and J. Burgemeister, "Piezoelectric Accelerometers Theory and Application,” 6th ed., Radebeul: Manfred Weber Metra Mess- und Frequenztechnik in Radebeul e.K., 2012.

[16] A. Sharma, F. M. Zaman, B. V. Amini and F. Ayazi, "A high-Q in-plane SOI tuning fork gyroscope,” IEEE SENSORS Conference, Vienna, Austria, 24-27 Oct. 2004.

[17] Q. Li, D. Xiao, X. Zhou, Z. Hou, Y. Xu and X. Wu, "Quality Factor Improvement in the Disk Resonator Gyroscope by Optimizing the Spoke Length Distribution," Journal of Microelectromechanical Systems, vol. 27, no. 3, pp. 414-423, June 2018.

[18] D. Xiao, X. Zhou, Q. Li, Z. Hou, X. Xi, Y. Wu and X. Wu, "Design of a Disk Resonator Gyroscope With High Mechanical Sensitivity by Optimizing the Ring Thickness Distribution,” Journal of Microelectromechanical Systems, vol. 25, no. 4, pp. 606-616, Aug. 2016.

[19] Y. Cheng, W. Zhang, J. Tang, D. Sun and W. Chen, "A MEMS piezoelectric solid disk gyroscope with improved sensitivity," Microsystem Technologies, vol. 21, pp. 1371-1377, 2015.

[20] Y. Wang, Q. Fu, Y. Zhang, W. Zhang, D. Chen, L. Yin, and X. Liu, “A Digital Closed-Loop Sense MEMS Disk Resonator Gyroscope Circuit Design Based on Integrated Analog Front-end," Sensors (MDPI), vol. 20, 2020.

[21] J. Xie, Y. Hao, W. Yuan, "Energy Loss in a MEMS Disk Resonator Gyroscope,” Micromachines (Basel), vol. 10, Jul 2019.

[22] H. Almabrouk, S. Kaziz, B. Mezghani, F. Tounsi and Y. Bernard, "Design Presentation and Induced-Stress Study of a 6-axis Single-Mass Piezoelectric IMU," 30th International Conference on Microelectronics (ICM), Sousse, Tunisia, 2018, pp. 144-147, 2018.

[23] H. Almabrouk, S. Kaziz, B. Mezghani, F. Tounsi and Y. Bernard, "Performance Enhancement of an Improved Design of 6-axis SingleMass Piezoelectric IMU," 30th International Conference on Microelectronics (ICM), Sousse, Tunisia, 2018, pp. 140-143, 2018.

[24] H. Almabrouk, B. Mezghani, G. Agnus, S. Kaziz, Y. Bernard, and F. Tounsi "Geometry Investigation and Performance Optimization of a Single-Mass Piezoelectric 6-DOF IMU”, Engineering, Technology \& Applied Science Research, vol. 10, pp. 6282-6289, October, 2020.

[25] F. Braghin, E. Leo and F. Resta, "The damping in MEMS inertial sensors both at high and low pressure levels,” Nonlinear Dynamics, vol. 54, pp. 79-92, Apr. 2008.

[26] H. Almabrouk, M. Hadj Said, B. Mezghani, F. Tounsi and Y. Bernard, "Simultaneous Angular and Linear Motion Detection Circuitry for a MEMS 6-DOF Piezoelectric IMU”, IEEE International Conference on Design \& Test of Integrated Micro \& Nano-Systems (DTS), Gammart, Tunisia, Avril, 2019.

[27] Y-H. Wang, P. Song, X. Li, C. Ru, G. Ferrari, P. Balasubramanian, M. Amabili, Y. Sun and X. Liu, "A Paper-Based Piezoelectric Accelerometer,” Micromachines (Basel), vol. 9, pp. 1-12, 2018.

[28] Z. Shen, C.Y. Tan, K. Yao, L. Zhang and Y. F. Chen, "A miniaturized wireless accelerometer with micromachined piezoelectric sensing element, " Sensors and Actuators A, Vol. 241, pp. 113-119, 2016.

[29] C-Y. Chang and T-L. Chen, "Design, Fabrication, and Modeling of a Novel Dual-Axis Control Input PZT Gyroscope,” sensors, vol. 11, 2017.

[30] E. Fujii, R. Takayama, K. Nomura, A. Murata, T. Hirasawa, A. Tomozawa, S. Fujii, T. Kamada, and H. Torii, "Preparation of (001) Oriented $\mathrm{Pb}(\mathrm{Zr}, \mathrm{Ti}) \mathrm{O} 3$ Thin Films and Their Piezoelectric Applications," IEEE Transactions on ultrasonics, ferroelectrics, and frequency control, vol. 54, no. 12, Dec. 2007.

[31] A. Parent, O.L. Traon, S. Masson and B.L. Foulgoc, "A Coriolis Vibrating Gyro Made of a Strong Piezoelectric Material," In Proceedings of IEEE Sensors, pp. 876-879, Atlanta, GA, USA, October 2007.

[32] H. Lv, L. Qin and J. Liu, "Principle Research on a Single Mass SixDegree-of-Freedom Accelerometer With Six Groups of Piezoelectric Sensing Elements," IEEE Sensors Journal, vol. 15, Issue 16, pp. 33013310, 2015. 